\title{
New Results from the REX Survey
}

\author{
A. Caccianiga, M.J.M. Marchã \\ Observatório Astronómico de Lisboa, Tapada da Ajuda, 1349-018, \\ Portugal \\ T. Maccacaro, A. Wolter, R. Della Ceca \\ Osservatorio Astronomico di Brera, via Brera 28, I-20121, Milan, Italy \\ I.M. Gioia \\ Istituto di Radio Astronomia del CNR, via Gobetti 101, Bologna, Italy
}

\begin{abstract}
The REX survey is an ongoing project aimed at the selection of new samples of QSOs and BL Lac objects. Spectroscopic identification of the $\sim 1600$ sources in the survey is in progress reaching, so far, about $40 \%$ of complete identification level. In this paper, the most recent results derived from the REX survey are briefly summarized.
\end{abstract}

\section{The REX Survey: Sampling the BL Lac Population}

The main scientific goal of the REX survey is the selection of new samples of AGNs, like radio-loud QSOs and BL Lac objects. One of the most interesting characteristics of the REX survey is that it has been designed to sample the BL Lac population more homogeneously than previous radio or X-ray surveys. The EMSS survey (Morris et al. 1991), for instance, has selected objects with a flat $\mathrm{X}$-ray-to-radio spectral index $\left(\alpha_{R X}<0.7\right.$, defined as High Energy Peaked BL Lacs, HBL) while the radio selected 1 Jy sample (Stickel et al. 1991) has found sources with $\alpha_{R X}>0.7$ (Low Energy peaked BL Lacs, LBLs). In Figure 1 the $\alpha_{R X}$ distribution of the BL Lacs discovered so far in the REX survey is compared to that of the BL Lacs in the EMSS and the $1 \mathrm{Jy}$ samples. The REX survey is clearly finding many objects with intermediate properties between HBLs and LBLs, thus suggesting that the presence of a dichotomy in the BL Lac class, often claimed in the literature, could be artificially created by selection effects.

\section{The XB-REX Sample: Studying the Cosmological Evolution}

A first completely identified sub-sample of 237 REXs has already been selected by imposing an X-ray flux limit greater than $4 \times 10^{-13} \mathrm{erg} \mathrm{s}^{-1} \mathrm{~cm}^{-2}$ and a magnitude (B) brighter than 20.5. The resulting sample (the X-ray Bright REX sample, XB-REX) has been used to estimate the cosmological evolution of emission 


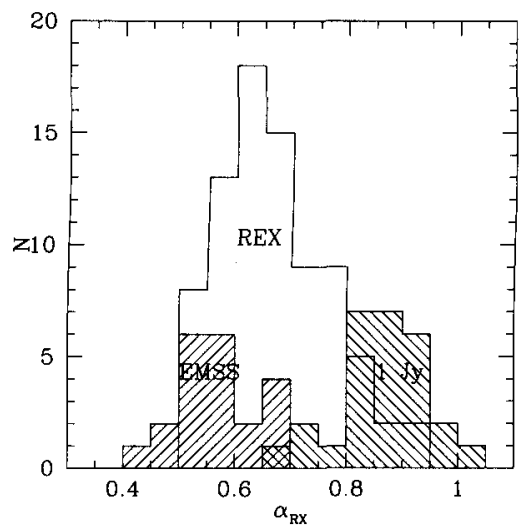

Figure 1. The $\alpha_{R X}$ distribution of the BL Lacs discovered so far in the REX, in the EMSS and in the $1 \mathrm{Jy}$ samples

line AGNs and BL Lac objects through a $V_{e} / V_{a}$ analysis. A detailed description of the results is presented in Caccianiga et al. (2001).

- BL Lacs. The XB-REX sample contains $55 \mathrm{BL}$ Lac objects and it is one of the largest complete samples of BL Lacs available for statistical studies. The $V_{e} / V_{a}$ test indicates that the population is not affected by a strong cosmological evolution. Moreover, the $V_{e} / V_{a}$ analysis applied to the subsample of HBLs does not reveal any significant departure from a uniform distribution. This result confirms that the cosmological properties of BL Lac objects are significantly different from those of emission line AGNs (see below).

- AGNs. The XB-REX sample contains $95 \mathrm{EL}$ AGN. The $V_{e} / V_{a}$ test is indicative of a strong positive evolution. We have then estimated the evolution by assuming a pure luminosity evolution of the form: $L(z)=L(0)(1+z)^{k}$ under the hypothesis that the values of $k$ in the three bands (radio, optical and X-ray) are the same. The best fit value is $k=3.0$ with a $1 \sigma$ interval of $(2.5,3.3)$. The value of $k$ is intermediate (but still consistent at $1 \sigma$ ) between that found for the X-ray selected ( $k=2.92$, Della Ceca et al. 1994) and the optically selected (e.g. $k=3.49$, Padovani 1993) radio-loud AGNs.

Acknowledgments. This work has received partial financial support from the Portuguese FCT and from the Italian Space Agency (ASI).

\section{References}

Caccianiga, A., et al. 2001, ApJ, in press

Caccianiga, A., et al. 1999, ApJ, 513, 51

Della Ceca, et al. 1994, ApJ, 430, 533

Morris, S.M., et al. 1991, ApJ, 380, 49

Padovani, P. 1993, MNRAS, 263, 461

Stickel, M., et al. 1991, ApJ, 374, 431 